Research Article

\title{
Novel Solution Method for Inventory Models with Stochastic Demand and Defective Units
}

\author{
Xu-Ren Luo ${ }^{1},{ }^{1}$ Chun-Hsiao Chu, ${ }^{2}$ and Henry C. J. Chao ${ }^{3}$ \\ ${ }^{1}$ Department of Computer Science and Information Engineering, Chung Cheng Institute of Technology, \\ National Defense University, Taoyuan, Taiwan \\ ${ }^{2}$ Department of Tourism, Aletheia University, Taipei, Taiwan \\ ${ }^{3}$ Department of Traffic Science, Central Police University, Taoyuan, Taiwan \\ Correspondence should be addressed to Xu-Ren Luo; nrgman.luo@gmail.com
}

Received 29 April 2020; Revised 24 August 2020; Accepted 19 September 2020; Published 10 October 2020

Academic Editor: Gordon Huang

Copyright (C) $2020 \mathrm{Xu}$-Ren Luo et al. This is an open access article distributed under the Creative Commons Attribution License, which permits unrestricted use, distribution, and reproduction in any medium, provided the original work is properly cited.

\begin{abstract}
This paper is a response to two papers. We improve the lengthy proof for the first paper by an elegant verification. For the second paper, we point out the three-sequence approach will result in different convergent rates such that when the other two sequences are converged, the ordering quantity sequence may still not converge to the optimal solution. We construct a novel iterative method to simplify the previous approach proposed by the three-sequence approach for the optimal solution. By the same numerical examples of three published papers, we demonstrate that we can control our findings to converge more accurately than previous results. Moreover, we show that there are three distinct features of our proposed approach. (i) It converges to the desired solution within the preassigned threshold value. (ii) We estimate the convergent ratio. (iii) We find the dominant factors for our proposed convergent sequence.
\end{abstract}

\section{Introduction}

For the past several decades, many important and interesting inventory models had been developed by researchers. For example, Rosenblatt and Lee [1] examined the relationship between imperfect quality of goods and lot size. Paknejad et al. [2] studied the connection between defective items and lead time for inventory models. Huang [3], Ouyang et al. [4], and $\mathrm{Wu}$ et al. [5] worked on inventory systems with the imperfect quality of goods or stochastic property of lead time. Wee et al. [6] focused on the interaction between the imperfect quality and shortages or nonshortages in his model. Chung [7] derived the necessary and sufficient constraints for the existence of the optimal solution for a single-vendor single-buyer integrated production-inventory problem under the condition of an unreliable process. Chung and Wee [8] constructed an integrated inventory model with warranty policy, tactical price, imperfect manufacture process, and inspection procedure. There are several researchers to explore the shortages during the lead time, for example, Das [9], Deng et al. [10], Ben-Daya and Raouf [11], Ouyang and $\mathrm{Wu}$ [12], and Wadhwa et al. [13]. Recently, there is a trend to improve previously published papers, for example, Deng [14], Deng et al. [15, 16], Lan et al. [17], Chang et al. [18], Jung et al. [19], and Tang et al. [20], that had provided useful analytical works to revise some questionable results in previous papers.

We will follow this trend to examine inventory models with stochastic demand, crashable lead time, and defective items that were developed by $\mathrm{Wu}$ and Ouyang [21], but they did not show the uniqueness of the optimal solution for their minimum cost problem. There are thirty-five papers that had cited $\mathrm{Wu}$ and Ouyang [21] in their references. We list them in the following: Mostard et al. [22], Wu et al. [5], Ho [23], Annadurai and Uthayakumar [24], Tung et al. [25], Çapar et al. [26], Drezner et al. [27], Lin et al. [28], Wang [29], Zheng and Liu [30], Gholami-Qadikolaei et al. [31], Ma and Qiu [32], Ouyang et al. [33], Lin [34-37], Parvathi et al. [37], Lin [38], Jindal and Solanki [39], Lin [40], Lin et al. [41], Vishkaei et al. [42], Wang et al. [43], Madhusoodhanan et al. 
[44, 45], Chang et al. [46], Jindal and Solanki [47], Madhusoodhanan et al. [48], Chang et al. [49], Sarkar and Mahapatra [50], Chuang et al. [51], Hu et al. [52], Maleki Vishkaei et al. [53], Noh et al. [54], and Singha et al. [55]. Most of them only mentioned Wu and Ouyang [21] in their introduction but did not offer further study for $\mathrm{Wu}$ and Ouyang [21].

On the contrary, Tung et al. [25] and Lin [38] provided a detailed examination for $\mathrm{Wu}$ and Ouyang [21] concerning the solution procedure of the iterative sequence whether or not they will converge to the optimal solution. Three conditions were obtained by Tung et al. [25] which are two upper bounds and one lower bound. They used a numerical examination to compare two upper bounds to decide the smaller one. Under their conditions, they merged the system of two first partial derivatives into a function of order quantity and verify the uniqueness of the optimal solution. Moreover, Tung et al. [25] criticized the iterative approach in $\mathrm{Wu}$ and Ouyang [21] to point out that the original development suggested by Wu and Ouyang [21] cannot be executed. And then Tung et al. [25] provided an example to illustrate that the results presented in $\mathrm{Wu}$ and Ouyang [21] did not match the optimal solution derived by Tung et al. [25] through a revised iterative approach proposed by Tung et al. [25]. Three papers had cited Tung et al. [25] in their references: Yang [56], Lin [38], and Hu et al. [50]. Two of them, Yang [56] and $\mathrm{Hu}$ et al. [50], mentioned Tung et al. [25] in their introduction, but did not provide any discussion for the solution approach proposed by Tung et al. [25]. Only one paper, Lin [38], constructed a new system that contains three convergent sequences to generate the desired sequence that converges to the optimal ordering quantity. Lin [38] further considered the iterative approach of Tung et al. [25] to point out that they did not verify the convergence of the iterative approach in their paper. She constructed three sequences to prove the convergence of them. Only one paper, $\mathrm{Hu}$ et al. [50], had cited Lin [38] in their reference. However, $\mathrm{Hu}$ et al. [50] did not provide any comments for the three-sequence approach proposed by Lin [38]. In this paper, firstly we will provide a simplified verification to replace the complicated proof proposed by Tung et al. [25]. Secondly, we develop a novel approach to generate a new sequence and then prove its convergence. Moreover, by the same numerical example of Tung et al. [25] and Lin [38], we show that our sequence converges better than that of Lin [38] so our findings are simple and yield better convergent result.

Several related papers concerning inventory models with defective items are worthy to mention: Khanna et al. [57], Gautam et al. [58], Gautam and Khanna [59], and Khanna et al. [60]. The organization of this paper is explained as follows. Section 2 provides notation and assumptions for the examined inventory model. Section 3 describes the results of $\mathrm{Wu}$ and Ouyang [21]. Section 4 reviews Tung et al. [25]. Section 5 discusses the findings of Lin [38]. Section 6 provides our new proof to verify that the solution for the first partial system is unique and is the optimal solution. Section 7 presents a numerical example to illustrate that our approach can obtain an optimal solution. Section 8 shows the three distinct features of our approach which is the key contribution of this paper. Section 9 concludes our paper.

\section{Notation and Assumptions}

We adopt the same notation and assumptions as $\mathrm{Wu}$ and Ouyang [21], Tung et al. [25], and Lin [38] and list them as follows:

\section{$D$ : expected demand per year}

A: setup cost per setup

$h$ : nondefective holding cost per unit per year

$h^{\prime}$ : defective holding cost per unit per year, $h^{\prime}<h$

$\pi$ : shortage cost per unit short

$\pi_{0}$ : marginal profit per unit

$v$ : unit inspection cost

$\beta$ : the fraction of the demand during the stock out period will be backordered, $0 \leq \beta \leq 1$

$p$ : the defective rate in an order lot (independent of lot size), $0 \leq p<1$, and it is a random variable

$g(p)$ : the probability density function (p.d.f.) of $p$

$f$ : the proportion of order quantity inspected

Q: lot size (order quantity), a decision variable

$L$ : length of the lead time, a decision variable

$X$ : the lead time demand with finite mean $\mu L$, and standard deviation $\sigma \sqrt{L}$ for lead time $L$

$r$ : reorder point, $r=\mu L+k \sigma \sqrt{L}$, where $k$ is the safety factor that is a decision variable

An arrival order may contain some defective items. We assume that the number of defective items in an arriving order of size $Q$ is a binomial random variable with parameters $Q$ and $p$, where $p(0 \leq p \leq 1)$ represents the defective rate in an order lot. Upon the arrival of an order, all the items are inspected and defective items in each lot will be returned to the vendor at the time of delivery of the next lot.

The inventory is continuously reviewed. Replenishments are made whenever the inventory level (based on the number of nondefective items) falls to the reorder point $r$.

The lead time $L$ has $n$ mutually independent components. The $i$ th component has a minimum duration $a_{1}$ and normal duration $b_{i}$, and a crashing cost per unit time $c_{i}$. Furthermore, for convenience, we rearrange $c_{i}$ such that $c_{1} \leq c_{2} \leq \cdots \leq c_{n}$.

The components of lead time are crashed one at a time starting with component 1 (because it has the minimum unit crashing cost) and then component 2 , etc.

If we let $L_{0}=\sum_{j=1}^{n} b_{j}$ and $L_{i}=\sum_{i+1}^{n} b_{j}+\sum_{j=1}^{i} a_{j}$, the lead time crashing cost $R(L)$ per cycle for a given $L \in\left[L_{i}, L_{i-1}\right]$ is given by $R(L)=c_{i}\left(L_{i-1}-L\right)+\sum_{j=1}^{i-1} c_{j}\left(b_{j}-a_{j}\right)$.

\section{Recap of Wu and Ouyang [21]}

$\mathrm{Wu}$ and Ouyang [21] constructed inventory models with stochastic demand, crashable lead time, and defective items. They applied the minimax distribution-free approach of 
Moon and Gallego [61] to develop the next minimum problem for variables: order quantity, $Q$, safety factor, $k$, and lead time $L$ :

$$
\begin{aligned}
\operatorname{EAC}^{u}(Q, k, L)= & \frac{\mathrm{AD}}{Q(1-E(p))}+\frac{h}{2}\left\{Q(1-E(p))+Q \frac{E\left(p^{2}\right)-E^{2}(p)}{1-E(p)}+\frac{E(p(1-p))}{1-E(p)}\right\} \\
& +h\left\{k \sigma \sqrt{L}+\frac{1-\beta}{2} \sigma \sqrt{L}\left(\sqrt{1+k^{2}}-k\right)\right\}+\frac{D\left(\pi+\pi_{0}(1-\beta)\right)}{2 Q(1-E(p))} \sigma \sqrt{L}\left(\sqrt{1+k^{2}}-k\right) \\
& +(Q-1) h^{\prime} \frac{E(p(1-p))}{1-E(p)}+\frac{\mathrm{Dv}}{1-E(p)}+\frac{D}{Q(1-E(p))}\left(c_{i}\left(L_{i-1}-L\right)+\sum_{j=1}^{i-1} c_{j}\left(b_{j}-a_{j}\right)\right)
\end{aligned}
$$

for $L \in\left[L_{i}, L_{i-1}\right]$, with $i=1, \ldots, n$.

$\mathrm{Wu}$ and Ouyang [21] proved that $\operatorname{EAC}^{u}(Q, k, L)$ concaves down for $L \in\left[L_{i}, L_{i-1}\right]$ such that the minimum will happen on the two boundary points $L_{i}$ or $L_{i-1}$. Consequently, under the restriction of $L=L_{i}$ or $L=L_{i-1}$, they derived the first partial derivatives for $Q$ and $k$.

To simplify the expression, Wu and Ouyang [21] used $L$ to represent $L=L_{i}$ or $L=L_{i-1}$ in the following derivations.

Using $(\partial / \partial Q) \operatorname{EAC}^{u}(Q, k, L)=0$ and $(\partial / \partial k) \operatorname{EAC}^{u}(Q, k$, $L)=0, \mathrm{Wu}$ and Ouyang [21] obtained that

$$
\begin{aligned}
Q= & {\left[\frac { 2 D } { h \delta } \left\{A+c_{i}\left(L_{i-1}-L\right)+\sum_{j=1}^{i-1} c_{j}\left(b_{j}-a_{j}\right)\right.\right.} \\
& \left.\left.+\frac{\pi+\pi_{0}(1-\beta)}{2} \sigma \sqrt{L}\left(\sqrt{1+k^{2}}-k\right)\right\}\right]^{(1 / 2)}, \\
& \frac{2 \sqrt{1+k^{2}}}{\sqrt{1+k^{2}}-k}=1-\beta+\frac{D\left(\pi+\pi_{0}(1-\beta)\right)}{h Q(1-E(p))},
\end{aligned}
$$

where

$$
\delta=1-2 E(p)+E\left(p^{2}\right)+2 \frac{h^{\prime}}{h} E(p(1-p)) .
$$

Nevertheless, the expressions in equations (2) and (3) and the variables $Q$ and $k$ are mixed. Hence, Wu and Ouyang [21] cannot verify the uniqueness of the optimal solution. On the contrary, Wu and Ouyang [21] mentioned that they will apply equations (2) and (3) to generate two sequences which will converge to the optimal solution as follows:

$$
\begin{aligned}
Q_{n+1}= & {\left[\frac { 2 D } { h \delta } \left\{A+c_{i}\left(L_{i-1}-L\right)+\sum_{j=1}^{i-1} c_{j}\left(b_{j}-a_{j}\right)\right.\right.} \\
& \left.\left.+\frac{\pi+\pi_{0}(1-\beta)}{2} \sigma \sqrt{L}\left(\sqrt{1+k_{n}^{2}}-k_{n}\right)\right\}\right]^{(1 / 2)}, \\
& \frac{2 \sqrt{1+k_{n+1}^{2}}}{\sqrt{1+k_{n+1}^{2}}-k_{n+1}}=1-\beta+\frac{D\left(\pi+\pi_{0}(1-\beta)\right)}{h Q_{n}(1-E(p))} .
\end{aligned}
$$

When $\beta=0$ and $L_{3}=3$, Wu and Ouyang [21] informed us that $Q^{*}=183$ without offering a detailed description for their solution procedure.

However, Tung et al. [25] pointed out that the results of $\mathrm{Wu}$ and Ouyang [21] did not match the reproduction examined by Tung et al. [25] which will be explained after Tables 1 and 2 of this paper.

\section{Review of Tung et al. [25]}

Based on the results of equations (2) and (3), Tung et al. [25] obtained that

$$
\begin{aligned}
& Q^{2}=\alpha_{1}+\alpha_{2}\left(\sqrt{1+k^{2}}-k\right), \\
& \frac{\sqrt{1+k^{2}}-k}{\sqrt{1+k^{2}}}=\frac{2 \alpha_{3} Q}{(1-\beta) \alpha_{3} Q+\alpha_{4}},
\end{aligned}
$$

where 
TABle 1: Reproduction Table 2 of Tung et al. [25]. Comparisons between $\mathrm{Wu}$ and Ouyang [21] and Tung et al. [25].

\begin{tabular}{ccccc}
\hline & \multicolumn{2}{c}{ Wu and Ouyang [21] } & \multicolumn{2}{c}{ Tung et al. [25] } \\
\hline$\beta$ & $Q$ & $\operatorname{EAC}^{u}(Q, k, L)$ & $Q$ & $\operatorname{EAC}^{u}(Q, k, L)$ \\
0 & 183 & 5697.95 & 179.74 & 5697.41 \\
\hline
\end{tabular}

TABle 2: Reproduction Table 4 of Tung et al. [25]. The revised iterative method of $\mathrm{Wu}$ and Ouyang [21] and executed by Tung et al. [25].

\begin{tabular}{lcccccc}
\hline & $n=1$ & $n=2$ & $n=3$ & $n=4$ & $n=5$ & $n=6$ \\
\hline$Q_{n}$ & 315.62 & 194.45 & 181.25 & 179.90 & 179.75 & 179.74 \\
$k_{n}$ & 2.2916 & 3.0248 & 3.1386 & 3.1510 & 3.1523 & 3.1524 \\
\hline
\end{tabular}

$$
\begin{aligned}
\alpha_{1} & =\left(\frac{2 D}{h \delta}\right)\left(A+c_{i}\left(L_{i-1}-L\right)+\sum_{j=1}^{i-1} c_{j}\left(b_{j}-a_{j}\right)\right), \\
\alpha_{2} & =\left(\frac{D}{h \delta}\right)\left(\pi+\pi_{0}(1-\beta)\right) \sigma \sqrt{L}, \\
\alpha_{3} & =h(1-E(p)), \\
\alpha_{4} & =D\left(\pi+\pi_{0}(1-\beta)\right),
\end{aligned}
$$

that satisfy $\alpha_{j}>0$, for $j=1, \ldots, 4$.

Based on equation (8), Tung et al. [25] obtained that

$$
1-\frac{\sqrt{1+k^{2}}-k}{\sqrt{1+k^{2}}}=1-\frac{2 \alpha_{3} Q}{(1-\beta) \alpha_{3} Q+\alpha_{4}},
$$

and then Tung et al. [25] simplified equation (13) to find that

$$
\frac{k}{\sqrt{1+k^{2}}}=\frac{\alpha_{4}-(1+\beta) \alpha_{3} Q}{(1-\beta) \alpha_{3} Q+\alpha_{4}} .
$$

Tung et al. [25] observed equation (14), under the condition that $k$ is the safety factor, with the condition $k \geq 0$. The solution for the first partial derivative system is an interior solution and then the restriction is modified from $k \geq 0$ to $k>0$.

Hence, Tung et al. [25] derived an upper bound for the ordering quantity, $Q$, as

$$
\alpha_{4}>(1+\beta) \alpha_{3} Q .
$$

We follow the solution approach of Tung et al. [25] to flip over equation (14) to derive that

$$
\frac{\sqrt{1+k^{2}}}{k}=\frac{(1-\beta) \alpha_{3} Q+\alpha_{4}}{\alpha_{4}-(1+\beta) \alpha_{3} Q}
$$

and then we take square on both sides of equation (16) to yield that

$$
\frac{1+k^{2}}{k^{2}}=\frac{\left[(1-\beta) \alpha_{3} Q+\alpha_{4}\right]^{2}}{\left[\alpha_{4}-(1+\beta) \alpha_{3} Q\right]^{2}} .
$$

We take "minutes one" from both sides of equation (17) to imply that

$$
\frac{1+k^{2}}{k^{2}}-1=\frac{\left[(1-\beta) \alpha_{3} Q+\alpha_{4}\right]^{2}}{\left[\alpha_{4}-(1+\beta) \alpha_{3} Q\right]^{2}}-1,
$$

and then we simplify equation (18) to find that

$$
\frac{1}{k^{2}}=\frac{4 \alpha_{3} Q\left(\alpha_{4}-\beta \alpha_{3} Q\right)}{\left[\alpha_{4}-(1+\beta) \alpha_{3} Q\right]^{2}} .
$$

After lengthy derivation, Tung et al. [25] obtained a relation to express $k$ as a function in the variable $Q$ as

$$
k=\frac{\alpha_{4}-(1+\beta) \alpha_{3} Q}{2 \sqrt{\alpha_{3} Q\left(\alpha_{4}-\beta \alpha_{3} Q\right)}} .
$$

Based on equation (20), Tung et al. [25] further derived that

$$
\begin{aligned}
\sqrt{1+k^{2}} & =\left(1+\frac{\left[\alpha_{4}-(1+\beta) \alpha_{3} Q\right]^{2}}{4 \alpha_{3} Q\left(\alpha_{4}-\beta \alpha_{3} Q\right)}\right)^{(1 / 2)} \\
& =\frac{\alpha_{4}+(1-\beta) \alpha_{3} Q}{2 \sqrt{\alpha_{3} Q\left(\alpha_{4}-\beta \alpha_{3} Q\right)}} .
\end{aligned}
$$

Plugging equations (20) and (21) into equation (7), then

$$
Q^{2}=\alpha_{1}+\alpha_{2} \sqrt{\frac{\alpha_{3} Q}{\alpha_{4}-\beta \alpha_{3} Q}} .
$$

Based on equation (22), Tung et al. [25] obtained a lower bound of $Q$ as

$$
Q>\sqrt{\alpha_{1}} .
$$

If we recall the inequality of equation (15), then

$$
\alpha_{4}-\beta \alpha_{3} Q>\alpha_{3} Q \text {. }
$$

Then, it follows that

$$
1>\frac{\alpha_{3} Q}{\alpha_{4}-\beta \alpha_{3} Q},
$$

that is,

$$
1>\sqrt{\frac{\alpha_{3} Q}{\alpha_{4}-\beta \alpha_{3} Q}} .
$$

Based on equation (26), Tung et al. [25] found another upper bound of $Q$ as

$$
Q<\sqrt{\alpha_{1}+\alpha_{2}} .
$$

Tung et al. [25] applied a numerical method to use data from $\mathrm{Wu}$ and Ouyang [21] to compare two upper bounds: $\alpha_{4} /(1+\beta) \alpha_{3}$ by equation (15) and $\sqrt{\alpha_{1}+\alpha_{2}}$ by equation (27). We cite Table 3 of Tung et al. [25] in the following.

Based on the results of Table 3, Tung et al. [25] observed that 
TABle 3: Reproduction Table 3 of Tung et al. [25]. The ratio of $\left(\alpha_{4} /(1+\beta) \alpha_{3} n \sqrt{\alpha_{1}+\alpha_{2}}\right)$.

\begin{tabular}{lcccc}
\hline & $L=L_{0}$ & $L=L_{1}$ & $L=L_{2}$ & $L=L_{3}$ \\
\hline$\beta=0$ & 18.855 & 20.090 & 21.856 & 22.962 \\
$\beta=0.5$ & 9.675 & 10.264 & 11.073 & 11.513 \\
$\beta=0.8$ & 6.211 & 6.552 & 6.994 & 7.180 \\
$\beta=1$ & 4.174 & 4.369 & 4.600 & 4.648 \\
\hline
\end{tabular}

$$
\frac{\alpha_{4}}{(1+\beta) \alpha_{3}}>\sqrt{\alpha_{1}+\alpha_{2}}
$$

Tung et al. [25] rewrote the rational function of equation (22) as follows:

$$
\left(Q^{2}-\alpha_{1}\right) \sqrt{\alpha_{4}-\beta \alpha_{3} Q}-\alpha_{2} \sqrt{\alpha_{3} Q}=0
$$

under the conditions

$$
\frac{\alpha_{4}}{(1+\beta) \alpha_{3}}>\sqrt{\alpha_{1}+\alpha_{2}}>Q>\sqrt{\alpha_{1}}
$$

with conditions of equations (9)-(12).

Based on equation (29), Tung et al. [25] assumed an auxiliary function:

$$
f(Q)=\left(Q^{2}-\alpha_{1}\right) \sqrt{\alpha_{4}-\beta \alpha_{3} Q}-\alpha_{2} \sqrt{\alpha_{3} Q} .
$$

Tung et al. [25] found that

$$
\begin{aligned}
& f^{\prime}(Q)=(2 Q) \sqrt{\alpha_{4}-\beta \alpha_{3} Q}+\left(Q^{2}-\alpha_{1}\right) \frac{-\beta \alpha_{3}}{2 \sqrt{\alpha_{4}-\beta \alpha_{3} Q}}-\frac{\alpha_{2} \sqrt{\alpha_{3}}}{2 \sqrt{Q}}, \\
& f^{\prime \prime}(Q)=\frac{2}{\sqrt{\alpha_{4}-\beta \alpha_{3} Q}}\left(\alpha_{4}-2 \beta \alpha_{3} Q\right)+\frac{\alpha_{2} \sqrt{\alpha_{3}}\left(\sqrt{\alpha_{4}-\beta \alpha_{3}}\right)^{3}-(\sqrt{Q})^{3}\left(\beta \alpha_{3}\right)^{2}\left(Q^{2}-\alpha_{1}\right)}{4\left(\sqrt{\left(\alpha_{4}-\beta \alpha_{3} Q\right) Q}\right)^{3}} .
\end{aligned}
$$
that

Tung et al. [25] obtained excellent derivations to claim

$$
\begin{aligned}
\alpha_{4} & \geq(1+\beta) \alpha_{3} Q \geq 2 \beta \alpha_{3} Q \\
\sqrt{\alpha_{3}}\left(\sqrt{\alpha_{4}-\beta \alpha_{3} Q}\right)^{3} & \geq \sqrt{\alpha_{3}}\left(\sqrt{\alpha_{3} Q}\right)^{3}=(\sqrt{Q})^{3}\left(\alpha_{3}\right)^{2} \\
& \geq(\sqrt{Q})^{3}\left(\beta \alpha_{3}\right)^{2} .
\end{aligned}
$$

Applying equations (33) and (34) with the inequality of equation (27), Tung et al. [25] proved the convexity property of $f(Q)$, that is,

$$
f^{\prime \prime}(Q)>0 \text {. }
$$

They further checked that

$$
\begin{aligned}
f\left(\sqrt{\alpha_{1}}\right)= & -\alpha_{2} \sqrt{\alpha_{1} \alpha_{3}}<0, \\
f\left(\sqrt{\alpha_{1}+\alpha_{2}}\right)= & \alpha_{2}\left[\alpha_{4}-(1+\beta) \alpha_{3} \sqrt{\alpha_{1}+\alpha_{2}}\right] \\
& \cdot\left[\sqrt{\alpha_{4}-\beta \alpha_{3} \sqrt{\alpha_{1}+\alpha_{2}}}+\sqrt{\alpha_{3}}\left(\alpha_{1}+\alpha_{2}\right)^{(1 / 4)}\right] .
\end{aligned}
$$
that

Based on the results of Table 3, Tung et al. [25] obtained

$$
f\left(\sqrt{\alpha_{1}+\alpha_{2}}\right)>0 \text {. }
$$

Hence, $f(Q)$ has a unique root for $\sqrt{\alpha_{1}+\alpha_{2}}>Q>\sqrt{\alpha_{1}}$ which satisfies the first-order partial derivatives of $(\partial / \partial Q) \operatorname{EAC}^{u}(Q, k, L)=0$ and $(\partial / \partial k) \operatorname{EAC}^{u}(Q, k, L)=0$, so it is the optimal solution of $\operatorname{EAC}^{u}(Q, k, L)$. On the contrary, Tung et al. [25] pointed out that the iterative approach proposed by $\mathrm{Wu}$ and Ouyang [21] was not consistent with their findings in the numerical examples.

Tung et al. [25] mentioned that given $k_{0}=0$ in equation (5), then $Q_{1}$ is obtained by the following formula:

$$
\begin{aligned}
Q_{1}= & {\left[\frac { 2 D } { h \delta } \left\{A+c_{i}\left(L_{i-1}-L\right)+\sum_{j=1}^{i-1} c_{j}\left(b_{j}-a_{j}\right)\right.\right.} \\
& \left.\left.+\frac{\pi+\pi_{0}(1-\beta)}{2} \sigma \sqrt{L}\left(\sqrt{1+k_{0}^{2}}-k_{0}\right)\right\}\right]^{(1 / 2)} .
\end{aligned}
$$

However, plugging $Q_{1}$ into equation (8), researchers only find that

$$
\frac{2 \sqrt{1+k_{1}^{2}}}{\sqrt{1+k_{1}^{2}}-k_{1}}=1-\beta+\frac{D\left(\pi+\pi_{0}(1-\beta)\right)}{h Q_{1}(1-E(p))}
$$

which is a relation containing $k_{1}$. However, researchers cannot directly derive $k_{1}$.

Therefore, Tung et al. [25] applied their finding of equation (20), together with equation (7) to generate the following approach:

$$
\begin{gathered}
Q_{n+1}=\sqrt{\alpha_{1}+\alpha_{2}\left(\sqrt{1+k_{n}^{2}}-k_{n}\right)}, \\
k_{n+1}=\frac{\alpha_{4}-(1+\beta) \alpha_{3} Q_{n}}{2 \sqrt{\alpha_{3} Q_{n}\left(\alpha_{4}-\beta \alpha_{3} Q_{n}\right)}} .
\end{gathered}
$$


For completeness, we reproduce Tables 2 and 4 of Tung et al. [25] to show that the findings of $\mathrm{Wu}$ and Ouyang [21] did not satisfy their claim.

From Table 2, Tung et al. [25] mentioned that if researchers execute the iterative method proposed by $\mathrm{Wu}$ and Ouyang [21], then the optimal ordering quantity, $Q^{*}=179.74$. However, $\mathrm{Wu}$ and Ouyang [21] claimed that $Q^{*}=183$. Hence, Tung et al. [25] asserted that the iterative method proposed by $\mathrm{Wu}$ and Ouyang [21], which is too complicated, cannot execute their method.

In Section 6, we will provide one simplification and another alternative approach to replace the lengthy solution procedure proposed by Tung et al. [25].

\section{Discussion of Lin [38]}

Lin [38] further examined the sequence convergent problem discussed by Wu and Ouyang [21] and Tung et al. [25]. In the following, we provide our comments for Lin [38]. Lin [38] admitted that Tung et al. [25] and improved the iterative approach of $\mathrm{Wu}$ and Ouyang [21], but Tung et al. [25] did not prove the convergence of their new approach. Hence, Lin [38] developed a new derivation that consists of three sequences as follows:

$$
Q_{n+1}=\left(B_{1}+B_{2}\left(\sqrt{1+k_{n}^{2}}-k_{n}\right)\right) \text {, }
$$

with

$$
\begin{aligned}
B_{1} & =\frac{2 D}{h \delta}\left[A+c_{i}\left(L_{i-1}-L\right)+\sum_{j=1}^{i-1} c_{j}\left(b_{j}-a_{j}\right)\right], \\
B_{2} & =\frac{2 D}{h \delta}\left[\frac{\pi+\pi_{0}(1-\beta)}{2}\right] \sigma \sqrt{L}, \\
d_{n+1} & =\frac{1}{2}\left(1-\beta+\frac{D\left[\pi+\pi_{0}(1-\beta)\right]}{h Q_{n+1}(1-E(p))}\right), \\
k_{n+1} & =\frac{d_{n+1}-1}{\sqrt{2 d_{n+1}-1}} .
\end{aligned}
$$

Moreover, Lin [38] proved the convergence of her approach. For interested readers, please refer to Lin [38] for her detailed proof. In the next section, we will develop our method which will be shown superior to that of Lin [38] which will be demonstrated by the example in Section 7 . Therefore, to save the precious space of this journal, we did not further discuss the detailed proof proposed by Lin [38] concerning the convergence of her three convergent sequence approach.

For later discussion, we cite results derived by Lin [38] in the following.

\section{Our Improved Approach}

Now, we begin to develop our verification for the uniqueness of the optimal ordering quantity to simplify the lengthy approach proposed by Tung et al. [25]. We observe (8) to imply that
TABLE 4: Reproduction from Lin [38]. For $\beta=0.5$ and $L_{3}=3$, the three sequences generated by Lin [38].

\begin{tabular}{cccc}
\hline$i$ & $k_{i}$ & $Q_{i}$ & $d_{i}$ \\
\hline 0 & 0 & - & - \\
1 & 1.925382 & 271.444019 & 8.884377 \\
2 & 2.433960 & 179.201796 & 13.328831 \\
3 & 2.490503 & 171.872873 & 13.886532 \\
4 & 2.495817 & 171.206597 & 13.939601 \\
5 & 2.496308 & 171.145237 & 13.944509 \\
6 & 2.496353 & 171.139579 & 13.944962 \\
7 & 2.496357 & 171.139060 & 13.945003 \\
8 & 2.496357 & 171.139014 & 13.945007 \\
9 & 2.496357 & 171.139014 & 13.945007 \\
\hline
\end{tabular}

$$
\frac{\sqrt{1+k^{2}}-k}{2 \sqrt{1+k^{2}}}=\frac{\alpha_{3} Q}{(1-\beta) \alpha_{3} Q+\alpha_{4}} .
$$

If $(A / B)=(C / D)$, then $(A /(-A+B))=(C /(-C+D))$. We apply this rule to equation (43) to derive that

$$
\frac{\sqrt{1+k^{2}}-k}{\sqrt{1+k^{2}}+k}=\frac{\alpha_{3} Q}{\alpha_{4}-\beta \alpha_{3} Q} \text {. }
$$

We know that

$$
\sqrt{1+k^{2}}-k=\frac{1}{\sqrt{1+k^{2}}+k} .
$$

Using equation (54), we find that

$$
\left(\sqrt{1+k^{2}}-k\right)^{2}=\frac{\alpha_{3} Q}{\alpha_{4}-\beta \alpha_{3} Q}
$$

If we plug our findings of equation (55) into equation (7), then we derive the results of equation (22) as proposed by Tung et al. [25]. Therefore, we demonstrate a simplified approach to find the same result as Tung et al. [25] without deriving $\sqrt{1+k^{2}}$ of equation (21) or finding $k$ of equation (20).

After simplifying the approach of Tung et al. [25], we will show our new solution method. Based on equation (8), we express $Q$ as a function in the variable $k$; then,

$$
Q=\frac{\left(\sqrt{1+k^{2}}-k\right) \alpha_{4}}{\alpha_{3}\left[(1+\beta) \sqrt{1+k^{2}}+(1-\beta) k\right]}
$$
that

We plug the findings of equation (47) into (7) to derive

$$
\left(\frac{\left(\sqrt{1+k^{2}}-k\right) \alpha_{4}}{\alpha_{3}\left[(1+\beta) \sqrt{1+k^{2}}+(1-\beta) k\right]}\right)^{2}=\alpha_{1}+\alpha_{2}\left(\sqrt{1+k^{2}}-k\right) \text {. }
$$

We rewrite equation (48) as 


$$
\begin{aligned}
& \left(\frac{\left(\sqrt{1+k^{2}}-k\right) \alpha_{4}}{\alpha_{3}\left[\beta\left(\sqrt{1+k^{2}}-k\right)+\left(1 / \sqrt{1+k^{2}}-k\right)\right]}\right)^{2} \\
& \quad=\alpha_{1}+\alpha_{2}\left(\sqrt{1+k^{2}}-k\right) .
\end{aligned}
$$

Therefore, our goal becomes to prove that there is a unique point that satisfies equation (49).

We observe equation (49) to find if we assume a new variable, denoted as $s$, where

$$
s=\sqrt{1+k^{2}}-k
$$

With the new variable, we rewrite equation (49) as follows:

$$
\alpha_{3}^{2}\left(\beta s^{2}+1\right)^{2}\left(\alpha_{1}+\alpha_{2} s\right)=\alpha_{4}^{2} s^{4} .
$$

Owing to equation (51), we assume an auxiliary function, denoted as $g(s)$, where

$$
g(s)=\alpha_{3}^{2}\left(\beta s^{2}+1\right)^{2}\left(\alpha_{1}+\alpha_{2} s\right)-\alpha_{4}^{2} s^{4} .
$$

We derive that

$$
g^{\prime}(s)=4 \beta \alpha_{3}^{2} s\left(\beta s^{2}+1\right)\left(\alpha_{1}+\alpha_{2} s\right)+\alpha_{2} \alpha_{3}^{2}\left(\beta s^{2}+1\right)^{2}-4 \alpha_{4}^{2} s^{3},
$$

$g^{\prime \prime}(s)=8 \beta^{2} \alpha_{3}^{2} s^{2}\left(\alpha_{1}+\alpha_{2} s\right)+4 \beta \alpha_{3}^{2}\left(\beta s^{2}+1\right)\left(\alpha_{1}+3 \alpha_{2} s\right)-12 \alpha_{4}^{2} s^{2}$,

$$
g^{\prime \prime \prime}(s)=24 \beta^{2} \alpha_{3}^{2} s\left(\alpha_{1}+2 \alpha_{2} s\right)+12 \beta \alpha_{2} \alpha_{3}^{2}\left(\beta s^{2}+1\right)-24 \alpha_{4}^{2} s,
$$

$$
g^{(4)}(s)=24 \beta^{2} \alpha_{3}^{2}\left(\alpha_{1}+5 \alpha_{2} s\right)-24 \alpha_{4}^{2}
$$

For the later discussion, we will begin to show that

$$
\begin{gathered}
3 \alpha_{1}>\alpha_{2}, \\
\alpha_{1}>0, \\
\alpha_{2}>0 .
\end{gathered}
$$

We recall $\alpha_{1}=(2 D / h \delta)\left(A+c_{i}\left(L_{i-1}-L\right)+\sum_{j=1}^{i-1} c_{j}\left(b_{j}-\right.\right.$ $\left.a_{j}\right)$ ) of equation (9), and from equation (10), we know that $\alpha_{2}=(D / h \delta)\left(\pi+\pi_{0}(1-\beta)\right) \sigma \sqrt{L}$ such that two inequalities of equations (58) and (59) are valid.

We will recall examples from $\mathrm{Wu}$ and Ouyang [23], Tung et al. [20], and Lin [13]. Hence, we adopt the same data as them with $D=600$ units/year, $A=200$ per order, $h=20$, $h^{\prime}=12, v=1.6, \pi=50, \pi_{0}=150$, and $\sigma=7$; the lead time has three components such that $L_{0}=8, R\left(L_{0}\right)=0, L_{1}=6$, $R\left(L_{1}\right)=5.6, L_{2}=4, R\left(L_{2}\right)=22.4, L_{3}=3$, and $R\left(L_{2}\right)=57.4$; the defective rate $p$ has a Beta distribution, with the probability density function $g(p)=4(1-p)^{3}$ to imply that $E(p)=(1 / 5)$; and $E\left(p^{2}\right)=(1 / 15)$, the fraction of the backordered demand, $\beta$, has four values, $0,0.5,0.8$, and 1 .
From the above data, we know that the lower bound $\sqrt{\alpha_{1}}=$ 136.683 and the upper bound $\sqrt{\alpha_{1}+\alpha_{2}}=271.444$.

Based on $\alpha_{1}=18.68 \times 10^{3}$ and $\alpha_{2}=54.99 \times 10^{3}$, we derive that

$$
3 \alpha_{1}-\alpha_{2}=1.05 \times 10^{3}>0 .
$$

Hence, the inequality of equation (57) is valid under data from $\mathrm{Wu}$ and Ouyang [21], Tung et al. [25], and Lin [38].

We recall that $s=\sqrt{1+k^{2}}-k$ of equation (50), with $k>0$, and then we derive that

$$
0<s<1 .
$$

Based on equations (58), (59), and (61), we find that

$$
\alpha_{2} s<\alpha_{1}+\alpha_{2} .
$$

Based on equation (28), we imply that

$$
\alpha_{4}^{2}>(1+\beta)^{2} \alpha_{3}^{2}\left(\alpha_{1}+\alpha_{2}\right),
$$

and then owing to $0 \leq \beta \leq 1$, we know that

$$
2 \beta \leq 1+\beta \text {. }
$$

We derive that

$$
\begin{aligned}
& g(0)=\alpha_{1} \alpha_{3}^{2}>0, \\
& g(1)=\alpha_{3}^{2}(1+\beta)^{2}\left(\alpha_{1}+\alpha_{2}\right)-\alpha_{4}^{2}<0,
\end{aligned}
$$

owing to equation (28). Next, we find that

$$
\begin{aligned}
g^{\prime}(0)= & \alpha_{2} \alpha_{3}^{2}>0, \\
g^{\prime}(1)= & 4 \beta \alpha_{3}^{2}(1+\beta)\left(\alpha_{1}+\alpha_{2}\right)+\alpha_{2} \alpha_{3}^{2}(1+\beta)^{2}-4 \alpha_{4}^{2} \\
= & 2\left[(2 \beta)(1+\beta) \alpha_{3}^{2}\left(\alpha_{1}+\alpha_{2}\right)-\alpha_{4}^{2}\right] \\
& +\left[\alpha_{2} \alpha_{3}^{2}(1+\beta)^{2}-\alpha_{4}^{2}\right]-\alpha_{4}^{2}<0 .
\end{aligned}
$$

And then, we compute that

$$
\begin{aligned}
g^{\prime \prime}(0)= & 4 \beta \alpha_{1} \alpha_{3}^{2}>0, \\
g^{\prime \prime \prime}(1)= & 8 \beta^{2} \alpha_{3}^{2}\left(\alpha_{1}+\alpha_{2}\right)+4 \beta \alpha_{3}^{2}(1+\beta)\left(\alpha_{1}+3 \alpha_{2}\right)-12 \alpha_{4}^{2} \\
= & 2\left[(2 \beta)^{2} \alpha_{3}^{2}\left(\alpha_{1}+\alpha_{2}\right)-\alpha_{4}^{2}\right] \\
& +2\left[(2 \beta)(1+\beta) \alpha_{3}^{2}\left(\alpha_{1}+3 \alpha_{2}\right)-3 \alpha_{4}^{2}\right]-4 \alpha_{4}^{2}<0 .
\end{aligned}
$$

Finally, we find that

$$
\begin{aligned}
g^{\prime \prime \prime}(0)= & 12 \beta \alpha_{2} \alpha_{3}^{2}>0, \\
g^{\prime \prime \prime}(1)= & 24 \beta^{2} \alpha_{3}^{2}\left(\alpha_{1}+2 \alpha_{2}\right)+12 \beta \alpha_{2} \alpha_{3}^{2}(1+\beta)-24 \alpha_{4}^{2} \\
= & 6\left[(2 \beta)^{2} \alpha_{3}^{2}\left(\alpha_{1}+2 \alpha_{2}\right)-2 \alpha_{4}^{2}\right] \\
& +6\left[(2 \beta)(1+\beta) \alpha_{2} \alpha_{3}^{2}-\alpha_{4}^{2}\right]-6 \alpha_{4}^{2}<0 .
\end{aligned}
$$
that 


$$
4\left(\alpha_{1}+\alpha_{2}\right)>\alpha_{1}+5 \alpha_{2}
$$

Hence, we derive that

$$
\alpha_{4}^{2}>4 \beta^{2} \alpha_{3}^{2}\left(\alpha_{1}+\alpha_{2}\right)>\beta^{2} \alpha_{3}^{2}\left(\alpha_{1}+5 \alpha_{2}\right) .
$$

Based on equation (74), we rewrite $g^{(4)}(s)$ of equation (56) to show that

$$
g^{(4)}(s)=24\left[\beta^{2} \alpha_{3}^{2}\left(\alpha_{1}+5 \alpha_{2} s\right)-\alpha_{4}^{2}\right]<0 .
$$

From equation (75), we know that $g^{(4)}(s)<0$, for $0<s<1$ and then $g^{(3)}(s)$ is a strictly increasing function. We recall that $g^{(3)}(0)>0$ of equation (71) and $g^{(3)}(1)<0$ of equation (72) such that we know that there is a point, denoted as $s^{\#}$, that satisfying

$$
g^{\prime \prime \prime}\left(s^{\#}\right)=0,
$$

with

$$
g^{\prime \prime \prime}(s)>0,
$$

for $0<s<s^{\#}$, and

$$
g^{\prime \prime \prime}(s)<0,
$$

for $s^{\#}<s<1$.

Based on our findings of equations (77) and (78), we derive that $g^{\prime \prime}(s)$ is an increasing function for $0<s<s^{\#}$, and $g^{\prime \prime}(s)$ is a decreasing function for $s^{\#}<s<1$.

Together with $g^{\prime \prime}(0)>0$ of equation (69) and $g^{\prime \prime}(1)<0$ of equation (70), we know that there is a point, denoted as $s^{\Delta}$ that satisfies $g^{\prime \prime}\left(s^{\Delta}\right)=0$ such that

$$
g^{\prime \prime}(s)>0
$$

for $0<s<s^{\Delta}$, and

$$
g^{\prime \prime}(s)<0,
$$

for $s^{\Delta}<s<1$.

Based on our findings of equations (79) and (80), we derive that $g^{\prime}(s)$ is an increasing function for $0<s<s^{\Delta}$, and $g^{\prime}(s)$ is a decreasing function for $s^{\Delta}<s<1$.

Together with $g^{\prime}(0)>0$ of equation (67) and $g^{\prime}(1)<0$ of equation (68), we know that there is a point, denoted as $s^{\Omega}$ that satisfies $g^{\prime}\left(s^{\Omega}\right)=0$ such that

$$
g^{\prime}(s)>0,
$$

for $0<s<s^{\Omega}$, and

$$
g^{\prime}(s)<0,
$$

for $s^{\Omega}<s<1$.

Based on our findings of equations (81) and (82), we derive that $g(s)$ is an increasing function for $0<s<s^{\Omega}$, and $g(s)$ is a decreasing function for $s^{\Omega}<s<1$.

Together with $g(0)>0$ of equation (65) and $g(1)<0$ of equation (66), we know that there is a point, denoted as $s^{*}$ that satisfies $g\left(s^{*}\right)=0$ such that

$$
g(s)>0
$$

for $0<s<s^{*}$, and

$$
g(s)<0,
$$

for $s^{*}<s<1$.

Based on the above discussion, we prove that there is a unique point that satisfies $0<s^{*}<1$, and

$$
g\left(s^{*}\right)=0 .
$$

Based on equation (50), we find that

$$
k^{*}=\frac{1-\left(s^{*}\right)^{2}}{2 s^{*}}
$$

satisfies equation (48).

We recall equation (47), and we find that

$$
Q^{*}=\frac{\left(\sqrt{1+\left(k^{*}\right)^{2}}-k^{*}\right) \alpha_{4}}{\alpha_{3}\left[(1+\beta) \sqrt{1+\left(k^{*}\right)^{2}}+(1-\beta) k^{*}\right]} .
$$

From the above discussion, we provide an alternative solution approach to show the uniqueness of the optimal solution.

Our proof needs an extra condition: $3 \alpha_{1}>\alpha_{2}$, which was supported by numerical examples in $\mathrm{Wu}$ and Ouyang [21], Tung et al. [25], and Lin [38]. Hence, we provide a simplified proof to replace the complicated verification in Tung et al. [25] with an extra condition of $\alpha_{4}>(1+\beta) \alpha_{3} \sqrt{\alpha_{1}+\alpha_{2}}$ to find an upper bound and a lower bound of the order quantity.

From equation (31), we rewrite $f(Q)=0$ to yield the following fifth-degree polynomial:

$$
\beta \alpha_{3} Q^{5}-\alpha_{4} Q^{4}-2 \beta \alpha_{1} \alpha_{3} Q^{3}+2 \alpha_{1} \alpha_{4} Q^{2}+\left(\beta \alpha_{1}^{2}+\alpha_{2}^{2}\right) \alpha_{3} Q-\alpha_{1}^{2} \alpha_{4}=0 .
$$

Based on equation (31), we derive that

$$
Q^{3}=\frac{2 \alpha_{1} \alpha_{4} Q^{2}+\left(\beta \alpha_{1}^{2}+\alpha_{2}^{2}\right) \alpha_{3} Q-\alpha_{1}^{2} \alpha_{4}}{Q\left(\alpha_{4}-\beta \alpha_{3} Q\right)+2 \beta \alpha_{1} \alpha_{3}} .
$$

Owing to the upper bound in equation (30), we know that $\alpha_{4}-\beta \alpha_{3} Q>0$, and the lower bound in equation (30) yields that $\alpha_{1} \alpha_{4} Q^{2}-\alpha_{1}^{2} \alpha_{4}>0$ such that the numerator and the denominator of equation (89) are both positive.

Motivated by equation (89), we will use the following relation:

$$
Q_{n+1}=\sqrt{\frac{2 \alpha_{1} \alpha_{4} Q_{n}^{2}+\left(\beta \alpha_{1}^{2}+\alpha_{2}^{2}\right) \alpha_{3} Q_{n}-\alpha_{1}^{2} \alpha_{4}}{Q_{n}\left(\alpha_{4}-\beta \alpha_{3} Q_{n}\right)+2 \beta \alpha_{1} \alpha_{3}}},
$$

to construct a sequence, $\left(Q_{n}\right)$, with an initial point $Q_{1}$. We will show that $\left(Q_{n}\right)$ is a convergent sequence. We evaluate $Q_{n+2}^{3}-Q_{n+1}^{3}$ and express the result as

$$
Q_{n+2}^{3}-Q_{n+1}^{3}=\left(Q_{n+1}-Q_{n}\right) \frac{N}{M},
$$


where

$$
\begin{aligned}
& N=y_{1}\left(Q_{n+1}+Q_{n}\right)+y_{3} Q_{n} Q_{n+1}+y_{4}>0, \\
& M=\Delta_{n} \Delta_{n+1}>0,
\end{aligned}
$$

with

$$
\begin{aligned}
& y_{1}=3 \beta \alpha_{1}^{2} \alpha_{3} \alpha_{4}, \\
& y_{2}=\beta \alpha_{3}^{2}\left(\beta \alpha_{1}^{2}+\alpha_{2}^{2}\right), \\
& y_{3}=2 \alpha_{1} \alpha_{4}^{2}+y_{2}, \\
& y_{4}=\alpha_{1}^{2} \alpha_{4}^{2}+2 \alpha_{1} y_{2}, \\
& \Delta_{n}=Q_{n}\left(\alpha_{4}-\beta \alpha_{3} Q_{n}\right)+2 \beta \alpha_{1} \alpha_{3} .
\end{aligned}
$$

Depending on the relation of $Q_{2}-Q_{1}$, our solution results are divided into three cases. Case (a) $Q_{2}-Q_{1}<0$; Case (b) $Q_{2}-Q_{1}=0$; Case (c) $Q_{2}-Q_{1}>0$.

For Case (a), owing to $Q_{2}-Q_{1}<0$ and equations (91)-(93), we know that $Q_{3}-Q_{2}<0$, and then $Q_{n+1}-Q_{n}<0$ for $n=1,2, \ldots$, such that $\left(Q_{n}\right)$ is an increasing sequence which is bounded above by $\sqrt{\alpha_{1}+\alpha_{2}}$, and then the increasing sequence will converge to its least upper bound.

For Case (b), from $Q_{2}=Q_{1}$, it yields that $Q_{3}=Q_{2}$ and $Q_{n+1}=Q_{n}$ for $n=1,2, \ldots$, such that $\left(Q_{n}\right)$ is a constant sequence that converges to $Q_{1}$.

For Case (c), from $Q_{2}-Q_{1}>0$ and equations (91)-(93), it yields that $Q_{3}-Q_{2}>0$, and then $Q_{n+1}-Q_{n}>0$ for $n=1,2, \ldots$, such that $\left(Q_{n}\right)$ is a decreasing sequence which is bounded below by $\sqrt{\alpha_{1}}$, and then the decreasing sequence will converge to its greatest lower bound.

Now, we summarize our results for three different cases to derive that our proposed sequence $\left(Q_{n}\right)$ will converge.

From our findings, we can claim that the optimal solution is between the limit of Case (a) and the limit of Case (c) such that depending on the accuracy of the optimal solution, we can obtain the optimal solution as accuracy as desired which will be demonstrated in the next section.

\section{Numerical Examples}

We will compare our findings with that of $\mathrm{Wu}$ and Ouyang [21], Tung et al. [25], and Lin [38]. Hence, we adopt the same data as them with $D=600$ units/year, $A=200$ per order, $h=20, h^{\prime}=12, v=1.6, \pi=50, \pi_{0}=150$, and $\sigma=7$; the lead time has three components such that $L_{0}=8, R\left(L_{0}\right)=0$, $L_{1}=6, \quad R\left(L_{1}\right)=5.6, \quad L_{2}=4, \quad R\left(L_{2}\right)=22.4, \quad L_{3}=3$, and $R\left(L_{3}\right)=57.4$; the defective rate $p$ has a Beta distribution, with probability density function $g(p)=4(1-p)^{3}$ to imply that $E(p)=(1 / 5)$; and $E\left(p^{2}\right)=(1 / 15)$, the fraction of the backordered demand, $\beta$, has four values, $0,0.5,0.8$, and 1 . From the above data, we know that the lower bound $\sqrt{\alpha_{1}}=$ 136.683 and the upper bound $\sqrt{\alpha_{1}+\alpha_{2}}=271.444$. Therefore, for Case (a), we select $Q_{1}=\sqrt{\alpha_{1}+\alpha_{2}}$ with $L=L_{3}=3$ and $\beta=0.5$ to derive our first numerical example. To save the precious space of the journal, we use different decimal places without influencing the tendency of the limit for our proposed decreasing sequence. We derive that $Q_{1}=271.4$, $Q_{2}=231.1, Q_{3}=191.6, Q_{4}=181.9, Q_{5}=177.0, Q_{6}=174.4$,
$Q_{7}=173.0, \quad Q_{8}=172.2, \quad Q_{9}=171.7, \quad Q_{10}=171.5$, $Q_{11}=171.33, Q_{12}=171.25, Q_{13}=171.20, Q_{14}=171.18$, $Q_{15}=171.16, Q_{16}=171.151, Q_{17}=171.146, Q_{18}=171.143$, $Q_{19}=171.1412, Q_{20}=171.1403, Q_{21}=171.1397, Q_{22}=$ $171.1394, \quad Q_{23}=171.1392, \quad Q_{24}=171.1391, \quad Q_{25}=$ $171.139085, Q_{26}=171.139052, Q_{27}=171.139033, Q_{28}=$ $171.139022, Q_{29}=171.139016, Q_{30}=171.139013, Q_{31}=$ $171.139010, Q_{32}=171.1390093, Q_{33}=171.1390087, Q_{34}=$ $171.1390083, Q_{35}=171.1390081, Q_{36}=171.1390080, Q_{37}=$ $171.13900793, Q_{38}=171.13900789, Q_{39}=171.13900786$, $Q_{40}=171.13900785, Q_{41}=171.139007844$, and $Q_{42}=$ 171.139007840 .

From the above sequence, if we decide to stop up to the eighth decimal place, we can claim that the optimal solution

$$
Q^{*} \leq 171.13900784 \text {. }
$$

For our second numerical example, we select $Q_{1}=\sqrt{\alpha_{1}}$ with $L=L_{3}=3$ and $\beta=0.5$ and then list our results for the increasing sequence as follows: $Q_{1}=136.7, Q_{2}=147.2$, $Q_{3}=155.6, Q_{4}=161.6, Q_{5}=165.4, Q_{6}=167.8, Q_{7}=169.2$, $Q_{8}=170.0, Q_{9}=170.5, Q_{10}=170.8, Q_{11}=170.9, \quad Q_{12}=$ 171.02, $Q_{13}=171.07, Q_{14}=171.10, Q_{15}=171.116, Q_{16}=$ $171.126, Q_{17}=171.132, Q_{18}=171.135, Q_{19}=171.1366$, $Q_{20}=171.1376, Q_{21}=171.1382, Q_{22}=171.13855, Q_{23}=$ $171.138746, Q_{24}=171.138858, Q_{25}=171.138922, Q_{26}=$ $171.138959, Q_{27}=171.138980, Q_{28}=171.138992, Q_{29}=$ $171.138999, Q_{30}=171.1390026, Q_{31}=171.1390048, Q_{32}=$ $171.1390061, Q_{33}=171.1390069, Q_{34}=171.1390073, Q_{35}=$ $171.13900751, Q_{36}=171.13900765, Q_{37}=171.13900773$, $Q_{38}=171.13900777, Q_{39}=171.1390077998$, and $Q_{40}=$ 171.1390078145 .

From the above sequence, if we decide to stop up to the eighth decimal place, we can state that the optimal solution

$$
Q^{*} \geq 171.13900781 \text {. }
$$

We have computed to the tenth decimal place to obtain that

$$
171.1390078339 \leq Q^{*} \leq 171.1390078341 .
$$

Because we have constructed a decreasing sequence with $Q_{1}=\sqrt{\alpha_{1}+\alpha_{2}}$ and an increasing sequence with $Q_{1}=\sqrt{\alpha_{1}}$, we can accurately estimate the optimal solution.

If we compare our findings with Tung et al. [25], then they derived that $Q^{*}=171.139009$ which was obtained by a decreasing sequence proposed by Tung et al. [25]. The result of Tung et al. [25] is consistent with our results when we check our decreasing sequence with $Q_{32}=171.1390093$ and $Q_{33}=171.1390087$ to express the finding to the sixth decimal place; then, we will stop at $Q^{*} \leq 171.139009$.

Furthermore, we compare our results with Lin [38]. She derived that $Q^{*}=171.139014$ which is found by a decreasing sequence proposed by Lin [38]. It indicates that the decreasing sequence $\left(Q_{i}\right)$ of Lin [38] did not converge to the desired result. The implicit reason for her unsuccessful converge may result from that there are three sequences in the iterative process of Lin [38]. We can predict that $\left(k_{i}\right)$ or $\left(d_{i}\right)$ converge faster than $\left(Q_{i}\right)$ so that the equal value to the sixth decimal place of $\left(k_{i}\right)$ or $\left(d_{i}\right)$ mislead the convergence 
for $\left(Q_{i}\right)$. The above observation supports that our derivation is better than the three-sequence approach of Lin [38]. Hence, our approach implies one-sequence is a good method. In the next section, we will explore three distinct characters proposed by our approach.

\section{Three Distinct Features of Our New Approach}

In this section, we show that there are three distinct features of our new approach.

The first feature is that we can derive the optimal solution within the preassigned threshold value. If we obtained a decreasing sequence, say $\left(x_{n}\right)$, with $x_{1}=271.4$ and an increasing sequence, say $\left(z_{n}\right)$, with $z_{1}=136.7$, the exact optimal solution is denoted as $Q^{*}$.

We know that

$$
z_{1}<z_{2}<\cdots<z_{n}<\cdots<Q^{*}<\cdots<x_{n}<\cdots<x_{2}<x_{1} .
$$

For a given threshold value, say $\varepsilon$, after we find a number, say $m$ that satisfies

$$
x_{m}-z_{m}<\varepsilon
$$

then we assume our optimal solution, denoted as $Q^{\Delta}$ with

$$
Q^{\Delta}=\frac{x_{m}+z_{m}}{2}
$$

such that we obtain

$$
z_{m}<Q^{*}<x_{m}
$$

We combine our findings of equations (99)-(101) to derive that

$$
\left|Q^{\Delta}-Q^{*}\right|<\varepsilon,
$$

to indicate our selected solution, $Q^{\Delta}$, is as close to the exact optimal solution, $Q^{*}$, within the preassigned threshold value.

The second feature is that we can estimate the converge ratio of our increasing sequence. Based on equation (91), we find that

$$
\frac{Q_{n+2}-Q_{n+1}}{Q_{n+1}-Q_{n}} \approx \frac{2 y_{1} Q^{*}+y_{3}\left(Q^{*}\right)^{2}+y_{4}}{3\left(Q^{*}\right)^{2}\left[Q^{*}\left(\alpha_{4}-\beta \alpha_{3} Q^{*}\right)+2 \beta \alpha_{1} \alpha_{3}\right]^{2}}
$$

From our results, we obtain that

$$
\frac{2 y_{1} Q^{*}+y_{3}\left(Q^{*}\right)^{2}+y_{4}}{3\left(Q^{*}\right)^{2}\left[Q^{*}\left(\alpha_{4}-\beta \alpha_{3} Q^{*}\right)+2 \beta \alpha_{1} \alpha_{3}\right]^{2}}=0.571840729 .
$$

We compute several related difference ratios and then list them in the following:

$$
\begin{aligned}
& \frac{Q_{29}-Q_{28}}{Q_{28}-Q_{27}}=0.571840853, \\
& \frac{Q_{30}-Q_{29}}{Q_{29}-Q_{28}}=0.571840779, \\
& \frac{Q_{31}-Q_{30}}{Q_{30}-Q_{29}}=0.571840770 .
\end{aligned}
$$

We compare results from equations (105)-(107) with our estimation of equation (104) to show that our estimation of equation (103) is very accurate when $n$ is big enough.

The third feature is that we can estimate the dominant factors to influence the convergence ratio proposed by our approach.

To estimate which factor influences the convergent ratio, we treat

$$
\alpha_{4}-\beta \alpha_{3} Q^{*} \approx \alpha_{4}
$$

such that we rewrite equation (104) as follows:

$$
\frac{y_{3}\left(Q^{*}\right)^{2}+2 y_{1} Q^{*}+y_{4}}{3\left(Q^{*}\right)^{2}\left[\alpha_{4} Q^{*}+2 \beta \alpha_{1} \alpha_{3}\right]^{2}}
$$

Based on

$$
\begin{aligned}
y_{3}\left(Q^{*}\right)^{2} & =6.16773 \times 10^{18}, \\
2 y_{1} Q^{*} & =2.15036 \times 10^{17}, \\
y_{4} & =1.97858 \times 10^{18}, \\
\alpha_{4} Q^{*} & =12835425.59, \\
2 \beta \alpha_{1} \alpha_{3} & =298916.129,
\end{aligned}
$$

we compare magnitudes of equations (110)-(114) to decide the dominant factors are (i) $Q^{*}$, (ii) $\alpha_{4}$, (iii) $y_{3}$, and (iv) $y_{4}$. Hence, we improve our findings of equation (103) to claim that

$$
\frac{Q_{n+2}-Q_{n+1}}{Q_{n+1}-Q_{n}} \approx \frac{y_{3}\left(Q^{*}\right)^{2}+y_{4}}{3 \alpha_{4}^{2}\left(Q^{*}\right)^{4}} .
$$

We compute that

$$
\begin{aligned}
& \frac{\left(y_{3}\left(Q^{*}\right)^{2}+y_{4} / 3 \alpha_{4}^{2}\left(Q^{*}\right)^{4}\right)}{\left(2 y_{1} Q^{*}+y_{3}\left(Q^{*}\right)^{2}+y_{4} / 3\left(Q^{*}\right)^{2}\left[Q^{*}\left(\alpha_{4}-\beta \alpha_{3} Q^{*}\right)+2 \beta \alpha_{1} \alpha_{3}\right]^{2}\right)} \\
& =98.412 \% .
\end{aligned}
$$

According to equation (116), we claim that our dominant factors can explain $98 \%$ of our convergent ratio.

We may provide a hypothetical example as follows: $Q_{1}=5.327, Q_{2}=2.049, Q_{3}=2.043$, and $Q_{4}=2.041$ with $\lim _{n \longrightarrow \infty} Q_{n}=1$. 
The researcher assumed that he will adopt the second decimal place to imply that $Q_{1}=5.33, Q_{2}=2.05, Q_{3}=2.04$, and $Q_{4}=2.04$.

Because the decimal expressions of $Q_{3}$ and $Q_{4}$ are the same, the researcher will accept that $Q^{*}=2.04$.

However, the convergent result at the second decimal place is not the optimal solution. The above example points out that depending on the convergence of the truncated decimal place without considering the convergent accuracy and ratio may not derive the optimal solution.

Our proposed approach can control the convergent accuracy and ratio that will be useful for future researchers to develop their convergent algorithms.

\section{Conclusion}

We follow the research trend to revise previously published papers of Wu and Ouyang [21], Tung et al. [25], and Lin [38]. Our approach simplifies the complex derivation in Tung et al. [25], and our generated sequence converges better than that of Lin [38]. At last, we show that our proposed method contained three distinct features. Our findings will provide a complete method for future papers in their proof for the existence and uniqueness of the optimal solution and explanation for their convergent environment.

\section{Data Availability}

The data of this inventory model are cited from $\mathrm{Wu}$ and Ouyang [21] which was published in Computers \& Industrial Engineering; Tung et al. [25] which was published in Abstract and Applied Analysis; and Lin [35] which was published in Abstract and Applied Analysis.

\section{Conflicts of Interest}

The authors declare that there are no conflicts of interest regarding the publication of this paper.

\section{Acknowledgments}

This research was partially supported by the Ministry of Science and Technology, with Grant no. MOST 106-2410-H156-009.

\section{References}

[1] M. J. Rosenblatt and H. L. Lee, "Economic production cycles with imperfect production processes," IIE Transactions, vol. 18 , no. 1 , pp. 48-55, 1986.

[2] M. J. Paknejad, F. Nasri, and J. F. Afflsco, "Defective units in a continuous review (s, Q) system," International Journal of Production Research, vol. 33, no. 10, pp. 2767-2777, 1995.

[3] C.-K. Huang, "An integrated vendor-buyer cooperative inventory model for items with imperfect quality," Production Planning \& Control, vol. 13, no. 4, pp. 355-361, 2002.

[4] L.-Y. Ouyang, K.-S. Wu, and C.-H. Ho, "Analysis of optimal vendor-buyer integrated inventory policy involving defective items," The International Journal of Advanced Manufacturing Technology, vol. 29, no. 11-12, pp. 1232-1245, 2006.
[5] K.-S. Wu, L.-Y. Ouyang, and C.-H. Ho, "Integrated vendorbuyer inventory system with sublot sampling inspection policy and controllable lead time," International Journal of Systems Science, vol. 38, no. 4, pp. 339-350, 2007.

[6] H. Wee, J. Yu, and M. Chen, "Optimal inventory model for items with imperfect quality and shortage backordering," Omega, vol. 35, no. 1, pp. 7-11, 2007.

[7] K.-J. Chung, "A necessary and sufficient condition for the existence of the optimal solution of a single-vendor singlebuyer integrated production-inventory model with process unreliability consideration," International Journal of Production Economics, vol. 113, no. 1, pp. 269-274, 2008.

[8] C. J. Chung and H.-M. Wee, "An integrated production-inventory deteriorating model for pricing policy considering imperfect production, inspection planning and warrantyperiod- and stock-level-dependant demand," International Journal of Systems Science, vol. 39, no. 8, pp. 823-837, 2008.

[9] C. Das, "Effect of lead time on inventory: a static analysis," Journal of the Operational Research Society, vol. 26, no. 2, pp. 273-282, 1975.

[10] P. S. Deng, R. H. J. Lin, J. Chang, S. T. Jung, and P. Chu, "Inventory Models with a negative exponential crashing cost taking time value into account," Journal of the Operations Research Society of Japan, vol. 51, no. 3, pp. 213-224.

[11] M. Ben-Daya and A. Raouf, "Inventory models involving lead time as a decision variable," The Journal of the Operational Research Society, vol. 45, no. 5, pp. 579-582, 1994.

[12] L.-Y. Ouyang and K.-S. Wu, "A minimax distribution free procedure for mixed inventory model with variable lead time," International Journal of Production Economics, vol. 5657, pp. 511-516, 1998.

[13] S. Wadhwa, K. S. Rao, and F. T. S. Chan, "Flexibility-enabled lead-time reduction in flexible systems," International Journal of Production Research, vol. 43, no. 5, pp. 3131-3162, 2005.

[14] P. S. Deng, "Improved inventory models with ramp type demand and Weibull deterioration," International Journal of Information and Management Sciences, vol. 16, no. 4, pp. 79-86, 2005.

[15] P. S. Deng, G. K. Yang, H. Chen, P. Chu, and D. Huang, "The criterion for the optimal solution of inventory model with stock-dependent consumption rate," International Journal of Information and Management Sciences, vol. 16, no. 2, pp. 97-109, 2005.

[16] P. S. Deng, C. Yen, C. Tung, Y. Yu, and P. Chu, “A technical note for the deteriorating inventory model with exponential time-varying demand and partial backlogging," International Journal of Information and Management Sciences, vol. 17, no. 2, pp. 101-108, 2006.

[17] C. Lan, Y. Yu, R. H. Lin, C. Tung, C. Yen, and P. S. Deng, “A note on the improved algebraic method for the EPQ model with stochastic lead time," International Journal of Information and Management Sciences, vol. 18, no. 1, pp. 91-96, 2007.

[18] S. K. J. Chang, H. L. Lei, S. T. Jung et al., "Note on deriving weights from pairwise comparison matrices in AHP," International Journal of Information and Management Sciences, vol. 19, no. 3, pp. 507-517, 2008.

[19] S. Jung, J. S. Lin, and J. P. C Chuang, "A note on "an EOQ model for items with Weibull distributed deterioration, shortages and power demand pattern"," International Journal of Information and Management Sciences, vol. 19, no. 4, pp. 667-672, 2008.

[20] D. W. Tang, H. C. Chao, and J. P. Chuang, "A note on the inventory model for deteriorating items with exponential 
declining demand and partial backlogging," International Journal of Information and Management Sciences, vol. 24, no. 2, pp. 167-173, 2013.

[21] K. S. Wu and L. Y. Ouyang, "Inventory model with defective items," Computers \& Industrial Engineering, vol. 39, no. 1-2, pp. 173-185, 2001.

[22] J. Mostard, R. De Koster, and R. Teunter, "The distributionfree newsboy problem with resalable returns," International Journal of Production Economics, vol. 97, no. 3, pp. 329-342, 2005.

[23] C. Ho, "A minimax distribution free procedure for an integrated inventory model with defective goods and stochastic lead time demand," International Journal of Information and Management Sciences, vol. 20, no. 1, pp. 161-171, 2009.

[24] K. Annadurai and R. Uthayakumar, "Controlling setup cost in (Q, r, L) inventory model with defective items," Applied Mathematical Modelling, vol. 34, no. 6, pp. 1418-1427, 2010.

[25] C. T. Tung, Y. W. Wou, S. W. Lin, and P. Deng, "Technical note on (Q, r, L) inventory model with defective items," Abstract and Applied Analysis, vol. 2010, Article ID 878645, 8 pages, 2010.

[26] İ. Çapar, B. Ekşioğlu, and J. Geunes, "A decision rule for coordination of inventory and transportation in a two-stage supply chain with alternative supply sources," Computers \& Operations Research, vol. 38, no. 12, pp. 1696-1704, 2011.

[27] Z. Drezner and O. Turel, "Normalizing variables with toofrequent values using a Kolmogorov-Smirnov test: a practical approach," Computers \& Industrial Engineering, vol. 61, no. 4, pp. 1240-1244, 2011.

[28] S.-W. Lin, Y.-W. Wou, and P. Julian, "Note on minimax distribution free procedure for integrated inventory model with defective goods and stochastic lead time demand," Applied Mathematical Modelling, vol. 35, no. 5, pp. 20872093, 2011.

[29] X. Wang, "Continuous review inventory model with variable lead time in a fuzzy random environment," Expert Systems with Applications, vol. 38, no. 9, pp. 11715-11721, 2011.

[30] H. Zheng and J. Liu, "Fuzzy random continuous review inventory model with imperfect quality," International Journal of Information and Management Sciences, vol. 22, no. 2, pp. 105-119, 2011.

[31] A. Gholami-Qadikolaei, M. Mohammadi, S. AmanpourBonab, and A. Mirzazadeh, "A continuous review inventory system with controllable lead time and defective items in partial and perfect lead time demand distribution information environments," International Journal of Management Science and Engineering Management, vol. 7, no. 3, pp. 205-212, 2012.

[32] W.-M. Ma and B.-B. Qiu, "Distribution-free continuous review inventory model with controllable lead time and setup cost in the presence of a service level constraint," Mathematical Problems in Engineering, vol. 2012, Article ID 867847, 16 pages, 2012.

[33] L.-Y. Ouyang, C.-T. Chang, and P. Shum, "The EOQ with defective items and partially permissible delay in payments linked to order quantity derived algebraically," Central European Journal of Operations Research, vol. 20, no. 1, pp. 141-160, 2012.

[34] H.-J. Lin, "An integrated supply chain inventory model with imperfect-quality items, controllable lead time and distribution-free demand," Yugoslav Journal of Operations Research, vol. 23, no. 1, pp. 87-109, 2013.

[35] H.-J Lin, "Controlling lost sales rate for inventory system with partial backlogging defective items," Journal of Applied Science and Engineering, vol. 16, no. 4, pp. 441-451, 2013.
[36] H.-J. Lin, "Reducing lost-sales rate on the stochastic inventory model with defective goods for the mixtures of distributions," Applied Mathematical Modelling, vol. 37, no. 5, pp. 32963306, 2013.

[37] S. Parvathi, R. Ramanarayanan, and S. Srinivasaraghavan, "General analysis of two products inventory system with seasonal production and sales," International Journal of Pure and Applied Mathematics, vol. 87, no. 2, pp. 195-204, 2013.

[38] J. Lin, "A study on iterative algorithm for stochastic distribution free inventory models," Abstract and Applied Analysis, vol. 2013, Article ID 251694, 3 pages, 2013.

[39] P. Jindal and A. Solanki, "Minimax distribution-free procedure for integrated inventory model with backorder price discount and controllable lead time," International Journal of Systems Science: Operations \& Logistics, vol. 1, no. 3, pp. 131-141, 2014.

[40] H.-J. Lin, "A collaborating inventory system in a supply chain under Brownian motion demand and imperfect quality," International Journal of Systems Science: Operations \& Logistics, vol. 1, no. 4, pp. 205-219, 2014.

[41] J. Lin, K.-C. Hung, and D. Tang, "Solution process for inventory models with crashable lead time," Applied Mathematics and Computation, vol. 227, pp. 335-340, 2014.

[42] B. M. Vishkaei, S. H. R. Pasandideh, and M. Farhangi, " $100 \%$ screening economic order quantity model under shortage and delay in payment," Scientia Iranica, vol. 21, no. 6, pp. 24292435, 2014.

[43] C. X. Wang, S. Webster, and S. Zhang, "Robust price-setting newsvendor model with interval market size and consumer willingness-to-pay," International Journal of Production Economics, vol. 154, pp. 100-112, 2014.

[44] P. Madhusoodhanan, P. Sekar, and R. Ramanarayanan, "Probabilistic analysis of general production and sales system with exponential machine time and manpower," Global Journal of Pure and Applied Mathematics, vol. 11, no. 2, pp. 601-610, 2015.

[45] P. Madhusoodhanan, P. Sekar, and R. Ramanarayanan, "General production and sales time with two-units system and manpower," International Journal of Applied Engineering Research, vol. 10, no. 23, pp. 43415-43421, 2015.

[46] C.-T. Chang, M.-C. Cheng, and P.-Y. Soong, "Impacts of inspection errors and trade credits on the economic order quantity model for items with imperfect quality," International Journal of Systems Science: Operations \& Logistics, vol. 3, no. 1, pp. 34-48, 2016.

[47] P. Jindal and A. Solanki, "Integrated supply chain inventory model with quality improvement involving controllable lead time and backorder price discount," International Journal of Industrial Engineering Computations, vol. 7, no. 3, pp. 463480, 2016.

[48] P. Madhusoodhanan, P. Sekar, and R. Ramanarayanan, "General production and sales time with two-unit system and Markovian manpower," Global Journal of Pure and Applied Mathematics, vol. 12, no. 1, pp. 31-40, 2016.

[49] C. Chang, P. Soong, and M. Cheng, "The influences of defective items and trade credits on replenishment decision," International Journal of Information and Management Sciences, vol. 28, no. 2, pp. 113-132, 2017.

[50] B. Sarkar and A. S. Mahapatra, "Periodic review fuzzy inventory model with variable lead time and fuzzy demand," International Transactions in Operational Research, vol. 24, no. 5, pp. 1197-1227, 2017.

[51] J. P. Chuang, S. S. Lin, and P. Ventriloquist, "Note on inventory model with reorder point as a decision variable," 
International Journal of Information and Management Sciences, vol. 29, no. 2, pp. 221-233, 2018.

[52] T. Hu, K. Hung, and K. Yang, "The convergence of Gallego's iterative method for distribution-free inventory models," Mathematics, vol. 7, no. 5, p. 10, 2019.

[53] B. Maleki Vishkaei, M. Seyyed-Esfahani, I. Mahdavi, and M. Askari, "A retailer inventory model when the reliability of inspection system affects the percentage of defective items which are delivered to final customers," Journal of Industrial and Production Engineering, vol. 36, no. 2, pp. 70-80, 2019.

[54] J. S. Noh, J. S. Kim, and B. Sarkar, "Stochastic joint replenishment problem with quantity discounts and minimum order constraints," Operational Research, vol. 19, no. 1, pp. 151-178, 2019.

[55] K. Singha, P. Parthanadee, and J. Buddhakulsomsiri, "Computational experiment of methods to determine periodic ( $R$, Q) inventory policy parameters: a case study of information decentralised distribution network," International Journal of Industrial and Systems Engineering, vol. 32, no. 2, pp. 212-242, 2019.

[56] G. K. Yang, "Analytical approach for optimal solution of inventory model with constrained production capacity," Concurrent Engineering, vol. 23, no. 1, pp. 74-80, 2015.

[57] A. Khanna, P. Gautam, B. Sarkar, and C. K. Jaggi, "Integrated vendor-buyer strategies for imperfect production systems with maintenance and warranty policy," RAIRO-Operations Research, vol. 54, no. 2, pp. 435-450, 2020.

[58] P. Gautam, A. Kishore, A. Khanna, and C. K. Jaggi, "Strategic defect management for a sustainable green supply chain," Journal of Cleaner Production, vol. 233, pp. 226-241, 2019.

[59] P. Gautam and A. Khanna, "An imperfect production inventory model with setup cost reduction and carbon emission for an integrated supply chain," Uncertain Supply Chain Management, vol. 6, no. 3, pp. 271-286, 2018.

[60] A. Khanna, P. Gautam, and C. K. Jaggi, "Coordinating vendor-buyer decisions for imperfect quality items considering trade credit and fully backlogged shortages," in Proceedings of the 2nd International Conference on Communication Systems-2015, B K Birla Institute of Engineering \& Technology, Pilani, Rajasthan, October 2015.

[61] I. Moon and G. Gallego, "Distribution free procedures for some inventory models," The Journal of the Operational Research Society, vol. 45, no. 6, pp. 651-658, 1994. 